\title{
Factors Associated with Sexual Intercourse Among African-Born Adolescents in Southern California
}

\author{
Dixon Anjejo, Naomi N. Modeste, Jerry W. Lee, Colwick M. Wilson \\ Loma Linda University
}

\begin{abstract}
Based on the theory of planned behavior the research aims were to: (a) ascertain how attitude, subjective norm, and perceived control predict intentions to engage in sexual intercourse among Black, African-born adolescents in Southern California; and (b) determine how behavioral beliefs, normative beliefs, and control beliefs predict attitude, subjective norm and perceived control. A questionnaire was developed based on focus group findings. One hundred and thirty-five adolescents aged 15 to 19 years completed the questionnaire. The strongest predictor of intention was attitude, then subjective norm. Enjoying sex, "wanting to be a parent” and to "keep sexual partners" predicted attitude. Educative, informative, cultural and faith based programs, if initiated may help African adolescents in Southern California.
\end{abstract}

(C)2007 Californian Journal of Health Promotion. All rights reserved.

Keywords: AIDS, Sexual intercourse, African born adolescents, Theory of planned behavior

The Joint United Nations Programme on HIV/AIDS (UNAIDS) (2006) estimates that there are about 40.3 million people living with HIV/AIDS worldwide. Five million are being infected every year and 25 million have died. The world is also raising about 14 million orphans and taking care of many critically ill people due to HIV/AIDS. Between 1,039,000 and 1,185,000 Americans were living with HIV in December 2003, with half of all the new cases reported among African Americans (Centers for Disease Control and Prevention [CDC], 2005a). This includes 180,000-280,000 people who do not know they are infected (CDC, 2005a). The American Foundation for AIDS Research in June 2005 reported that 1.5 million Americans have been infected with HIV since the start of the AIDS epidemic, and more than 524,000 have died of the disease (Quin and Overbaugh, 2005). Each year in the USA, about 20,000 young people are newly infected with HIV, and nearly four million new sexually transmitted infections occur among 15- to 19-year-old youth (Feijoo, 2004). Since the epidemic began, an estimated 10,041 adolescents with AIDS have died, and according to a CDC report (CDC, 2005b), the proportion with an AIDS diagnosis among adolescents and young adults increased from $3.9 \%$ in 1999 to $4.7 \%$ in 2003.

While African Americans constitute about 13\% of the US population, they account for $56 \%$ of "late testers" (those who missed opportunities in prevention and treatment of HIV). Many are youth aged 15 to 19 who are diagnosed with AIDS. More than half of the new HIV infections occur through heterosexual sex among African Americans younger than 25 years, and many adolescents infected with HIV are not aware of their infection. According to De Santis and Patsdaughter (2005), African American adolescents account for more than $60 \%$ of new cases of the disease in their age group, and more than $60 \%$ of children with HIV/AIDS are African Americans. Official reports on HIV/AIDS among African Americans do not differentiate African-born Black Africans from African Americans. Didier (2002) contends that this acts as a barrier for reaching these communities because the assumption is that they have the same risks.

The State of California has about 133,858 reported cases of AIDS, which is the second highest number of AIDS cases in the USA. 
Many of the cases of the disease in the state are reported in Southern California, which consists of San Bernardino, Riverside, Imperial, San Diego, Orange, Los Angeles, Ventura, Kern, Santa Barbara, and San Luis Obispo Counties. The County of San Diego 2004 Annual Report (County of San Diego, 2004) indicated that 12,034 of those cases are among San Diego residents, making the county the third largest contributor to AIDS prevalence in California, after Los Angeles and San Francisco. Many of the new cases reported in Southern California are among people of color. African Americans account for about $13 \%$ of AIDS cases that are reported in San Diego County. Since the data are not disaggregated by country of origin, AIDS cases from African-born Blacks are included.

Black Africans who are born in Africa and have moved to the US come from a continent which has just $10 \%$ of the world's population but has $73 \%$ of the world's HIV/AIDS cases (UNAIDS, 2006). In 2004, approximately 3.1 million new HIV infections occurred in Sub-Saharan Africa (Boer and Mashamba, 2005). About 26 million Africans in the same region are estimated to be HIV positive (UNAIDS, 2006). HIV/AIDS has become a disease affecting 8.6 million youth in Africa because the spread of the disease in this region is faster than in any other parts of the world. The spread is partly due to cultural beliefs, poverty, and severe lack of information and services for prevention (Ateka, 2001). One of the beliefs common among the youth in Africa is that AIDS is a disease for the "high risk group." They associate the disease with homosexuals, drug users, prostitutes, truck drivers and tourists. As a result, many youth discount risk to themselves because they do not identify with these high-risk groups (Akwara, Madise and Hinde, 2003).

The risk perception among the youth in South Africa is very low. They do not feel vulnerable, and $62 \%$ of those who learn they are HIV positive believe they face little or no risk at all of contracting the disease or getting AIDS (HIV/AIDS and Sexual and Reproductive Health, August, 2004). African youth are more likely to have unprotected sex. In many rural villages in Africa, people regard HIV/AIDS as a taboo subject for discussion in the community. It is unclear how African migrants in the United States perceive the risk of HIV/AIDS in their new or adopted country.

Reid (1999) also reported that youth in Africa grow up with a belief that men have stronger sexual drives than women, and men cannot live without sex. This belief has led many young people to have multiple sexual partners, with boys reported as being about seven times more sexually active than girls in the same age range of 15 to 19 years (Erulkar, Karueru, Kaggwa, Kingola, Nyagah, Ochieng, 1998; National Council for Population and Development [NCPD], 1999). Dube (2005) reported that some male South African students say AIDS is an acronym for "American Ideas of Discouraging Sex.” This notion drives African youth to initiate sexual intercourse at an early age. In Kenya, for example, a very large percentage of youth, about $90 \%$, are sexually active by age 20 . Girls and women, in general, lack the power to negotiate for safer sex because their sexual behavior is dictated by the societal sexual norms and practices (Akwara et al., 2003). In many communities in Africa, it is taboo for the children to discuss sexual matters with or in the presence of their parents (Fitzgerald and Fitzgerald, 1987). Even with the existing abundant knowledge from health educators and other sources regarding risks of unprotected sexual intercourse, many men often prefer having sexual intercourse with young girls because of the cultural belief that sex with a virgin will cure sexually transmitted diseases, including HIV/AIDS.

Unprotected sexual intercourse among adolescents continues to contribute significantly to population growth, high birth rates, and escalating rates of AIDS cases in many countries of sub-Saharan Africa. About one-third of African boys and girls ages 12 to 17 have had sexual intercourse, and one in five reports having their first sexual experience at the age of 12 or younger. There are also indications that in many Black sub-Saharan countries, the median age of adolescents of first intercourse is 16.5 years (Simbayi, Charveau, Shisana, 2004). According to Karungari and Zabin (1995), about 
$80 \%$ of African youth have engaged in risky sexual intercourse by age 18 and only $10 \%$ abstain from sex before marriage. Adolescents in the southern part of Africa are sexually active by age 15 years with some reporting up to seven partners, yet few take precautionary measures against sexually transmitted diseases and HIV/AIDS. In a South African national study (Shisana, 2002), 23\% of male adolescents aged $15-24$ and $8.8 \%$ of female adolescents reported concurrent sexual partnerships. Another study in the rural areas of Transkei in South Africa by Buga (1996), found mean ages of the girls and boys were 15 and 16 years, respectively. Seventy six percent of the girls and $90 \%$ of the boys had already engaged in sexual intercourse. Some of the factors that influence the median age at first intercourse in some countries of Africa include residence and level of education. For example, in Kenya, rural young women engage in intercourse earlier than urban women, and the median age at first intercourse for women with no education is three years earlier than women with at least a secondary school education. Population Reference Bureau and Population Service International (2000) conducted a formative research study in eight sub-Saharan African countries to identify and understand major barriers to delayed sexual debut among the African adolescents. Seventy four focus group discussions were conducted with both genders aged 14 to 19 years, sexually or not sexually active. The results indicated that the most important factor influencing adolescents' decision to have sexual intercourse was the desire to be a man or a woman (desire to make transition into adulthood). Other additional factors included peer pressure, sexual violence, coercion, and lack of communication between parents and children (Hartell, 2005; Profile Social Marketing and Communications for Health, February 2006).

According to Dunkle et al. (2004) HIV infection rates among teenage women are about five times higher than the rates for men throughout subSaharan Africa. For example, in Kenya, one teenage boy as compared to four teenage girls live with HIV. This happens because the physical immaturity of younger women and women's lower status in many African societies may prevent them from having control of their sexual relationships. Studies on women's first sexual intercourse experience showed that more than a half of young women in Malawi and 20\% of young women in Nigeria experienced forced sexual intercourse (UNAIDS, 1998; UNAIDS, 2000). Research shows that adolescents who start having sexual intercourse early are more likely to have sex with high-risk partners or multiple partners, and are less likely to use condoms.

When drugs are used in social situations which usually involve peers, adolescents are more exposed to voluntarily or involuntarily engage in unplanned acts of sexual intercourse and unprotected sex (Morojele, Brooks, \& Kachieng'a, 2006).

While much work has been done on determinants of risky behaviors in Africa, similarly much work has been done with African Americans, but little is known regarding Black adolescents born in Africa and living in the United States. Summit Health Institute for Research and Education 2005 reports that "very little is known about immigrants of African origin in California, and no population-based survey has focused comprehensively on their identification or their clinical and social needs (p. 6).” Kamya (1997) contends that studies regarding immigrants in the US have mostly focused on Hispanic, Asian and Russian immigrants. This study may help health professionals to better understand this minority community and aid in the development of appropriate programs tailored to their needs.

The purpose of this study was to determine factors associated with spread of AIDS among Black African born adolescents living in Southern California, and if cultural beliefs regarding sexual intercourse are salient factors contributing to the spread of AIDS among Black African born adolescents living in Southern California. Culture includes knowledge, belief, art, morals, law, custom, and any other capabilities and habits acquired by a human being as a member of society. Collectivist beliefs are one element of cultural beliefs. Collectivist beliefs are common in most 
countries of the African continent. Many people in different societies in Africa have a very strong attachment to their cultural beliefs and practices; they perform these cultural practices in order to be accepted by society and the community. These actions override any concerns about HIV/AIDS. For example, girls of Ugandan tribes pressure themselves to be pregnant to prove their fertility before marriage and boys prove their manhood in the community by impregnating girls, therefore, they tend to have unprotected sexual intercourse (Hulton, Cullen, \& Khalokho, 2000).

In collectivist cultures, individuals are more concerned with the opinion of the family and friends than in individualistic cultures (Aggleton, 1996; Airhihenbuwa \& Obregon, 2000). Thus, it seems reasonable to suppose that subjective norms, which are based on the opinions of other people (Ajzen, 2002), will be more important in collectivist cultures than in individualistic cultures. Are cultural factors major contributors to age of first sexual initiation among African-born adolescents living in Southern California?

This study was conducted in Southern California because a larger number of African-born immigrants, migrants and refugees live in this region than in any other part of California. According to Summit Health Institute for Research and Education (SHIRE), as of March 2005, Southern California had about 102,655 residents of African origin in California, with Los Angeles and San Diego Counties reporting 37,000 and 12,000 , respectively. In a recent health survey conducted among Africans who migrated to the United States and are residents of Los Angeles, Orange, San Bernardino and San Diego Counties, the respondents expressed dissatisfaction at the low level of cultural competence exhibited by their health care providers. The respondents believed that health care workers are very ignorant about African immigrants and their cultures (SHIRE, 2005). How these migrants access health care for HIV/AIDS or what their attitudes and beliefs are about the disease, and which risk behaviors are practiced are poorly understood. Therefore, we attempted to help understand how cultural beliefs and practices may influence the spread of HIV/AIDS among this migrant group.

\section{Theoretical Framework}

The theory of planned behavior (Ajzen, 1991) was used as a theoretical model for this study. In this theory, Ajzen (2002) indicates that the best predictors of teen's AIDS risk behaviors are their intentions. Intentions measure the extent to which the teen is motivated to initiate sexual intercourse at an early age and use a condom when having sex. The intentions are in turn determined by their attitudes towards the risky behavior, and the subjective norms, which is the participant's perception of social pressure to engage (or not) in AIDS risk behavior. Attitude represents the teen's feelings and beliefs about AIDS risk behaviors. Perceived behavioral control (PCB), which is perceived ease of performing or not performing the risky behavior, is the third component which determines the behavioral intentions. The teen's behavioral beliefs consisting of their beliefs about the outcomes of a behavior and their evaluation of those outcomes influence their attitudes.

Normative beliefs, which are beliefs about the expectations of others close to them and motivation to comply with the expectations, influence subjective norms. In this study normative beliefs are those that adolescents have about the parents' communication and guidance regarding the AIDS risky behaviors and the teen's motivation to comply with the parents' expectations. The teen's control beliefs beliefs about availability of resources, and opportunities necessary to achieve the behavior and how each of the resources and opportunities facilitate the behavior — influence their perceived control. The general rule is that the more favorable the attitude and the subjective norm and the greater the perceived control, the stronger should be the teen's intention to perform the risky behavior.

\section{Method}

\section{Study Design}

This study used a non-experimental crosssectional design (Cook \& Campbell, 1979) to investigate factors associated with sexual intercourse at an early age and HIV/AIDS risk 
behaviors among African born adolescents in Southern California, utilizing the theory of planned behavior as the theoretical framework (Ajzen, 2002). The study was conducted in two phases using both qualitative and quantitative methods. In the qualitative stage an open-ended questionnaire was administered to three focus groups to ascertain salient beliefs underlying risk behaviors associated with sexual intercourse. The first group consisted of ten adolescents. Each of the other two groups was composed of eight adolescents. Convenience samples were used for both the focus groups and the survey in the quantitative phase.

\section{Participants}

Participants in both phases of the study consisted of Black African born adolescents aged 15 to 19 years old who live in Southern California $(n=$ 135). For inclusion in the study, participants must have been born in Africa, and lived in the US for 10 years or less whether or not they were immigrants, migrants or undocumented African families. Only those who were willing to participate and met the conditions regarding age, years lived in the United States and country of birth were included in the study. Adolescents between 15 and 17 years old who met all the conditions and were willing to participate, but did not have parental consent were excluded from the study. However, participants who lived in the US for more than 10 years but less that 15 years and met the other inclusion criteria were included in the data analysis, in an effort to reach the required sample size.

\section{Procedure}

Recruitment for this study took place at churches and mosques where African born adolescents attended worship services, sport centers they frequented, and Non Governmental Organizations (NGOs) which deal with refugee settlements in Southern California. In San Diego we used the Sudanese American Presbyterian Church and Southern Sudanese Community Center as the recruitment and data collection sites for the Sudanese community. Somali Community Center and Horn of Africa Community Center were the sites used for Somali refugees who have settled in San Diego. Other centers included Project BRO of Project
Concern International, and Crafton High School in San Diego. The East African Community of Orange was the site for recruiting Ethiopians. Recruitment flyers were distributed through the recruitment centers one week or two before the data were collected. Flyers were posted in strategic centers that Black African born teens frequently visited, inviting youth $15-19$ years old to participate in the study. There were times when recruitment and data collection had to be done simultaneously especially in churches where the parents were present to sign the consent forms for their adolescents.

\section{Study Variables}

All variables in this study were based on selfreport from respondents who participated in the focus groups and completed the survey questionnaire. The dependent variable was the risk behavior, which was defined as initiation of sexual intercourse at an early age. The independent variables included attitude, subjective norms and perceived control. Also included in this category were demographic variables such as age, number of years lived in Southern California, gender, education level, country of origin, and religion, which were all necessary for defining the study population.

\section{Development of Instrument}

Development of the instruments involved qualitative and quantitative phases. The first was qualitative which used open-ended questions to assess possible salient factors related to sexual intercourse, using focus groups. Salient issues or topics were identified based upon the number of times a topic was mentioned in the discussion.

Also included were open-ended questions on behavioral beliefs on proof of fertility, multiple sexual partners, gender issues, normative beliefs about parental communication and guidance, and control beliefs. For the quantitative phase, a survey instrument was developed based on the results of the focus group questions and components of the theory of planned behavior as described by Ajzen (2002).

Qualitative questionnaire. The questions for the focus groups included the following areas: 
1. Outcome beliefs: beliefs about expected outcomes when participants engage in sexual intercourse and their evaluation of the outcomes. The questions were: (a) If you were to have sexual intercourse at your age what good things might happen? (b) Are there any other things you associate with your having sexual intercourse at your age?

2. Normative beliefs: questions in this section included the following (a) "Are there particular people who would approve of you having sexual intercourse at your age?” (b) "Are there particular people who would not approve of you having sexual intercourse at your age?” (c) Are there particular people who may or may not approve of you having sexual intercourse at your age?"

3. Control beliefs, included: (a) When you think of having sexual intercourse at your age what resources or opportunities would you need to help you do the behavior? (b) What resources or opportunities do you lack that might prevent you from having sexual intercourse at your age?

All the responses from the focus groups were transcribed and a quantitative questionnaire was developed based on the results. The theory of planned behavior was used as the theoretical framework.

Quantitative questionnaire. This questionnaire was divided into seven sections as follows: (a) Demographic characteristics of the respondents. Included in this category were age, gender, and country of origin, years lived in Southern California, religion, and education level. (b) Other questions were based on the theory of planned behavior. The Ajzen (2002) format of constructing the questionnaire was adapted and used in this section. Behavioral intention, attitude, subjective norm, and perceived control are the components of the theory of planned behavior that were used to develop the questionnaire. There were other sections that included outcomes, values, what others think about sexual intercourse at an early age, and motivation to comply with what others think. The last section included questions on availability of resources and the power of those resources to help in avoiding sexual intercourse at the participants' age. The questions were developed as outlined below:

Intention. Likert-scale questions with a range of 1 (strongly disagree) to 7 (strongly agree) were constructed to measure respondent's intention to initiate sexual intercourse in the next twelve months.

Attitude. Questions on attitude toward AIDS risk behaviors and outcomes when one has sex in the next twelve months. These questions were also designed using a Likert Scale format where there was a range of 1 (bad, risky, harmful and foolish) to 7 (good, not risky, beneficial and wise) respectively.

Subjective norm. There were questions on participants' perceptions regarding social pressure from the people they considered important to them such as parents, peer, siblings, and teachers as relates to the respondents engaging in sexual intercourse at their age. Responses were rated on a 7-point scale $(1=\mathrm{I}$ should not, $7=\mathrm{I}$ should and $1=$ extremely unlikely, 7 = extremely likely).

Perceived behavioral control. Perceived behavioral control, was perceived ease of engaging or not engaging in sexual intercourse within the next twelve months. The participants responses were rated on a 7 -point scale as follows: ( 1 = difficult, 7 = easy); (1= definitely false, $7=$ definitely true) and (1= strongly agree, $2=$ strongly disagree).

Other scales included the following:

Outcomes. This section asked about their perceptions on how likely certain outcomes were to happen if they engaged in sexual intercourse. Their responses were rated as ( $1=$ definitely would not happen, $7=$ definitely would happen).

Values. This section inquired of their perception of values that they attached to outcomes of their involvement in sexual intercourse. The responses were rated as ( $1=$ extremely bad, $7=$ extremely good). 


\section{Perception on others thoughts and motivation} to comply. These were questions dealing with their perceptions regarding social pressure from the people they considered important to them such as parents, peer, siblings, and teachers as related to engaging in sexual intercourse at their age. Participants responses were rated on a 7point scale $(1=$ I should not, $7=$ I should and $1=$ extremely unlikely, 7 = extremely likely).

Availability of resources and access to the resources: Questions on perceived behavioral control aimed at measuring the availability or access to resources and the power of those resources to help them avoid sexual intercourse at their age. Participants responses were rated on a 7-point scale as follows: access $(1=$ strongly disagree to $7=$ strongly agree that there is access to resources) and for power the scale of measurement ranged from (1= much difficult, to $7=$ much easier) to help the respondents avoid sexual intercourse at their age.

\section{Data Collection}

Qualitative phase. The first phase, which included the use of focus groups, was done on two visits in three different venues namely; Sudanese Presbyterian Church, a Somalia community center and Horn of Africa Center. The group discussions were scheduled for Sunday and Friday, days when the adolescents attend church with their parents and also come together to socialize at their community centers. After the investigator explained to each congregation the purpose and importance of the research, focus groups were formed based on gender. Each participant aged 18 or 19 years signed consent forms while parents or guardians consented for those aged 15 - 17 years. Then an open-ended questionnaire was administered to each participant in the three focus groups to ascertain salient beliefs underlying risk behaviors. The investigator presided over each focus group discussion forum and gave each participant equal opportunity to make contributions to the discussion. The discussions were taped and later transcribed. The tapes were destroyed after transcription. The first two focus groups consisted of five adolescents each. Each of the other two groups was composed of eight adolescents. Convenience samples were used for all the focus groups. At the end of the focus group session each participant received $\$ 10.00$ for participating in the discussion.

Quantitative phase. Data were collected over an eight-month period. The days and times of appointments followed the pattern of focus group phase except when there were special appointments in different venues such as schools. After consenting to be in the study, each participant aged 18 or 19 years completed the survey. For anonymity, they were asked not to write their names on the questionnaire. Parents or guardians of participants whose ages ranged from 15 to 17 years signed the consent forms on behalf of their children, and they assented to complete the survey. Participants were instructed on how to complete the questionnaire, and the benefits and risks, if any, were explained. Each participant was given up to thirty minutes to complete the questionnaire and after its completion, each received $\$ 10.00$ for participating. The completed questionnaires were then placed in an envelope until time for data entry.

\section{Statistical Analyses}

The Statistical Package for the Social Sciences (SPSS) version 13 was chosen for data entry and analysis. Descriptive statistics including frequencies, percentages, means, standard deviation and distributions were done on demographical variables. Scatter plots were diagramed to check for outliers. Alpha reliabilities for the theory of planned behavior constructs were calculated. Simple products of the variables were calculated without using optimal scaling technique and results are discussed in the appropriate sections.

\section{Results}

Demographic characteristics of participants are presented in Appendix A. The respondents' ages ranged from 15 to 19 years old, the majority of whom were males (61.5\%) and 38.5\% females. The majority of the participants have lived in Southern California for 5 to 10 years (79.3\%). Most were Muslims (70.4\%), followed by protestant Christians (24.45\%). Many of the participants were drawn from refugees of Somalia (59\%), Sudanese origin (22\%) and 
currently living in San Diego. Ethiopian participants came from Orange County with a few Christians from the Inland Empire. Over $74 \%$ were high school attendees with only $15 \%$ attending local community colleges.

The alpha reliability values for intention, attitude, subjective norm and perceived behavioral control were $0.63,0.87,0.61$ and 0.64 , respectively. All the reliability test results were within the acceptable range.

The perceived likelihood and perceived value of various sexual intercourse outcomes for both male and female respondents are given in Table 1. Generally, there were significant differences regarding what males and females thought about outcomes of having sexual intercourse, Pillai's Trace $=.188, F(11,123)=2.59, p=0.005$. However, according to the means, the likelihood of these adolescents having sex in the next twelve months were low, closer to "definitely would not happen," ranging from 3.06 to 4.09 for male participants and even lower for female participants . Likewise, there were significant differences regarding the perceptions of the male and females on the values of the outcome if they engaged in sexual intercourse, Pillai's Trace $=$ $.1160, \mathrm{~F}(11,123)=2.12, \mathrm{p}=0.023$. The mean for valuing outcomes for the same respondents followed a similar trend (2.87 to 4.08). On average, enjoying sex was perceived as a more likely outcome for male respondents when compared to females $\mathrm{t}(133)=2.79, \mathrm{p}=0.006$. Male respondents' perception regarding value for enjoying sex was also significantly higher than female respondents' perception, $t(133)=2.90, p=0.005$. Further, regarding likely outcomes, the males respondents' perception about "partner would stay" was also significantly higher than the female respondents' perception $\mathrm{t}(133)=2.16, \mathrm{p}=0.033$. It is very interesting to note that the respondents' average perception regarding "getting AIDS" when they have sex is that it would not happen or was unlikely that it would happen. However, as shown in Table 1 they perceived "getting AIDS" as an extremely bad outcome, if it happens as indicated in means of 2.87.

Table 1

Perceived likelihood and value for various sexual intercourse outcomes for both female and male respondents.

\begin{tabular}{|l|c|c|c|c|c|c|c|c|}
\hline & \multicolumn{4}{|c|}{ Male (n=83) } & \multicolumn{4}{c|}{ Female (n=52) } \\
\hline \multicolumn{1}{|c|}{ Outcomes } & \multicolumn{2}{|c|}{ Likelihood } & \multicolumn{2}{c|}{ Value } & \multicolumn{2}{c|}{ Likelihood } & \multicolumn{2}{c|}{ Value } \\
\hline & $M$ & $S D$ & $M$ & $S D$ & $M$ & $S D$ & $M$ & $S D$ \\
\hline Enjoying Sex & 4.09 & 2.41 & 4.08 & 2.46 & 2.94 & 2.28 & 2.85 & 2.38 \\
\hline Partner would stay & 3.92 & 2.22 & 3.29 & 2.05 & 3.03 & 2.37 & 2.81 & 2.25 \\
\hline Feeling guilty & 3.83 & 2.29 & 2.9 & 1.97 & 4.27 & 2.52 & 2.77 & 2.33 \\
\hline Peers acceptance & 3.54 & 2.07 & 3.28 & 2.06 & 2.87 & 2.29 & 2.55 & 2.07 \\
\hline Bad reputation & 3.53 & 2.22 & 2.89 & 2.06 & 4.52 & 2.45 & 2.71 & 2.36 \\
\hline Spreading rumors & 3.48 & 2.22 & 3.14 & 2.11 & 4.52 & 2.55 & 2.60 & 2.25 \\
\hline Unwanted baby & 3.48 & 2.27 & 2.76 & 2.10 & 4.13 & 2.61 & 2.05 & 1.94 \\
\hline Feeling good & 3.36 & 2.21 & 2.98 & 2.16 & 2.81 & 2.39 & 2.71 & 2.37 \\
\hline Get other diseases & 3.33 & 2.23 & 2.85 & 2.03 & 3.40 & 2.45 & 2.27 & 2.07 \\
\hline Proud parent & 3.28 & 2.21 & 3.08 & 2.11 & 2.66 & 2.26 & 2.60 & 2.24 \\
\hline Get AIDS & 3.06 & 2.14 & 2.87 & 2.09 & 3.79 & 2.33 & 1.87 & 1.70 \\
\hline
\end{tabular}

Note: Ranges for likelihood are: definitely won't happen (1) to definitely would happen (7) and for value are extremely bad (1) to extremely good (7). 
On the other hand, adolescent girls perceived that "bad reputation," "spreading rumors," "feeling guilty" and "getting unwanted baby" were generally the most likely outcomes if they engaged in sexual intercourse at their age. In fact, compared to males, females had more negative beliefs than males about "bad reputation" as a likely outcome if they engaged in sex $\mathrm{t}(133)=-2.37, \mathrm{p}=0.02$. Likewise, compared to males, females perceived "spreading rumors" as a more likely outcome of engaging in sexual intercourse $t(133)=-2.43$, $\mathrm{p}=0.02$, and there was nothing the female respondents perceived as significantly good in any of the outcomes. They generally perceived all outcomes of sexual intercourse as bad with "getting AIDS" as extremely bad followed by "getting an unwanted baby."
Table 2 presents the means of what the male and female respondents believed important people in their lives thought about them having sexual intercourse at their age and their motivation to comply with what others thought.. In general there were clearly significant differences regarding what males and females thought referents wanted them to do about having sexual intercourse, Pillai's Trace $=.282, \quad F(15$, $119)=3.12, p=0.0003$. Examination of separate ttests suggested that females were more likely to think their referents wanted them to avoid sexual intercourse than did males for every referent except mother, boy friend, and God. For example, compared to males, females believed their classmates were more negative towards the notion of them having sex, $\mathrm{t}(133)=3.66$, $\mathrm{p}=0.001$.

Table 2

Whether others think the participants should have sexual intercourse and the respondents' motivation to comply with what others want for both (male and female respondents).

\begin{tabular}{|l|c|c|c|c|c|c|c|c|}
\hline & \multicolumn{4}{|c|}{ Male (n=82) } & \multicolumn{4}{c|}{ Female (n=53) } \\
\hline & Normative Belief & \multicolumn{2}{|c|}{$\begin{array}{c}\text { Motivation to } \\
\text { comply }\end{array}$} & $\begin{array}{c}\text { Normative } \\
\text { Belief }\end{array}$ & \multicolumn{2}{c|}{ Motivation to comply } \\
\hline & Mean & SD & Mean & SD & Mean & SD & Mean & SD \\
\hline Classmate & 3.73 & 2.30 & 3.89 & 2.37 & 2.38 & 2.04 & 3.94 & 2.70 \\
\hline Girlfriend & 3.65 & 2.37 & 3.94 & 2.45 & 2.45 & 2.22 & 4.17 & 2.71 \\
\hline Friends & 3.42 & 2.21 & 3.83 & 2.32 & 2.08 & 1.99 & 4.08 & 2.71 \\
\hline Brother & 3.36 & 2.33 & 3.49 & 2.38 & 1.56 & 1.42 & 4.17 & 2.75 \\
\hline Cousin & 3.26 & 2.24 & 3.75 & 2.37 & 1.77 & 1.76 & 4.35 & 2.66 \\
\hline Boyfriend & 2.91 & 2.22 & 3.30 & 2.36 & 2.81 & 2.46 & 4.08 & 2.71 \\
\hline God & 2.87 & 2.35 & 3.67 & 2.49 & 2.11 & 2.18 & 4.27 & 2.81 \\
\hline Church members & 2.86 & 2.16 & 3.76 & 2.37 & 1.62 & 1.57 & 4.25 & 2.70 \\
\hline Teacher & 2.84 & 2.21 & 3.84 & 2.40 & 1.85 & 1.82 & 4.19 & 2.71 \\
\hline Grandfather & 2.80 & 2.15 & 3.69 & 2.43 & 1.65 & 1.61 & 4.33 & 2.80 \\
\hline Sister & 2.74 & 2.09 & 3.76 & 2.37 & 1.82 & 1.80 & 4.35 & 2.73 \\
\hline Religious Leader & 2.68 & 2.10 & 3.65 & 2.38 & 1.71 & 1.77 & 4.31 & 2.68 \\
\hline Mother & 2.63 & 2.08 & 3.72 & 2.57 & 1.96 & 1.95 & 4.25 & 2.85 \\
\hline Father & 2.60 & 2.11 & 3.53 & 2.59 & 1.69 & 1.70 & 4.13 & 2.81 \\
\hline Grandmother & 2.56 & 2.05 & 3.60 & 2.46 & 1.67 & 1.59 & 4.25 & 2.80 \\
\hline Nond
\end{tabular}

Note: ranges of Subjective Norm are I should not (1) to I should (7) and for wanting to do or not at all (1) and very much (7). 
The means of normative beliefs were very low, being below the midpoint 4 and ranging from 2.56 to 3.73 with standard deviation also ranging from 2.05 to 2.37. Overall results indicated that male respondents believed that important people in their lives thought they (the male respondents) should not have sex at their age. Leading in this category were probably grandmother, father, mother and religious leaders. However, these male participants seemed to believe that classmates, a girlfriend, good friends, brother, and cousins, presumably exerted no social pressure regarding their sexual behavior, or having sex at an early age. On the other hand, on average, males also seemed to be quite neutral on whether or not they wanted to comply with what important people in their lives thought and the mean for motivation to comply ranged from 3.60 to 3.94 .

The results of multivariate analysis indicated that there were non-significant differences between both male and female respondents regarding motivation to comply with what the referents thought about them (the respondents) having sexual intercourse. The general belief of the female respondents was that virtually all important people in their lives thought that they should not engage in sex at their age. The important people whom they listed were brother, church members, grandfather, grandmother, religious leaders, cousin, sister, teacher, then mother. Like the male respondents, the average female respondent also did not want to comply with what they believed their referents wanted them to do.

Table 3 shows the male and female respondents beliefs regarding availability of resources and the power of those resources to predict their perceived control on having sexual intercourse. Results from multivariate analysis indicated that there were non-significant differences between the means of males and females beliefs about availability of resources. However, there were significant differences regarding what males and females believed about available resources to help them have control over having sexual intercourse, Pillai's Trace $=.236, F(18,116)=$
1.996, $\mathrm{p}=0.015$. Generally, both male and female participants agreed that the resources they needed to help them have control over engaging in sexual intercourse at their age were available. The range of means for males' beliefs about available resources ranged from 3.77 to 4.55. The top three readily available resources were "proper education", "be well informed" and "avoid watching bad TV programs". It is also important to note that the male respondents neither believed the availability of parental talk nor denied the absence of such a talk as indicated in Table 3. It was apparent that male participants also believed the most powerful resources to help them have control and avoid sexual intercourse at their age were the following in their respective orders of their means: being strong in religious beliefs; avoiding watching bad television programs and visiting or attending a program on parenting (like a parenting center). Their thoughts on the rest of the resources were generally neutral. However, the least powerful resources were: talking to mother, talking to brother and talking to father.

Table 3 further shows how female respondents were even more enthusiastic in their thoughts or perceptions about availability of resources and power of those resources to help them avoid having sexual intercourse at their age. The means for the females' perception regarding availability of resources ranged from 4.17 to 5.48 , and their beliefs on power of the available resources ranged from 4.02 to 5.27. In their perception, females felt the most available resources included the following: talking to sister, having a house, avoiding watching bad television programs, a car, proper education, sharing social life with friends, being well informed and being strong in religious beliefs. But like men, the female respondents were relatively indifferent about the availability of the relatives, particularly parents being available to help. Females also perceived that religious beliefs, personal responsibility and church are the most powerful resources that could help them. 
Table 3

Perceived availability of resources needed to avoid having sexual intercourse and the perceived power of each resource to help.

\begin{tabular}{|l|c|c|c|c|c|c|c|c|}
\hline & \multicolumn{2}{|c|}{ Availability } & \multicolumn{2}{c|}{ Power to Help } & \multicolumn{2}{c|}{ Availability } & \multicolumn{2}{c|}{ Power to Help } \\
\hline \multicolumn{1}{|c}{ Resource } & Mean & SD & Mean & SD & Mean & SD & Mean & SD \\
\hline Proper education & 4.55 & 2.11 & 3.86 & 2.33 & 5.15 & 2.42 & 4.71 & 2.52 \\
\hline Be well informed & 4.53 & 2.25 & 3.90 & 2.26 & 5.09 & 2.46 & 4.68 & 2.52 \\
\hline In touch with culture & 4.52 & 2.28 & 3.92 & 2.28 & 5.35 & 2.35 & 4.87 & 2.54 \\
\hline Get a job & 4.49 & 2.28 & 4.00 & 2.32 & 5.17 & 2.25 & 4.98 & 2.26 \\
\hline Be strong in beliefs & 4.45 & 2.38 & 4.57 & 2.36 & 5.46 & 2.30 & 5.27 & 2.32 \\
\hline Money & 4.44 & 2.27 & 3.99 & 2.43 & 4.17 & 2.54 & 4.02 & 2.65 \\
\hline Be more responsible & 4.43 & 2.13 & 3.67 & 2.41 & 5.48 & 2.29 & 5.12 & 2.40 \\
\hline Listen to church & 4.42 & 2.29 & 3.88 & 2.43 & 4.92 & 2.49 & 5.02 & 2.50 \\
\hline Bad TV programs & 4.39 & 2.28 & 4.44 & 2.32 & 5.07 & 2.35 & 4.94 & 2.42 \\
\hline Talk to my brothers & 4.24 & 2.14 & 3.55 & 2.22 & 4.29 & 2.53 & 4.37 & 2.57 \\
\hline Parents' Guidance & 4.20 & 2.48 & 3.70 & 2.40 & 4.37 & 2.72 & 4.29 & 2.76 \\
\hline Get a car & 4.17 & 2.28 & 3.93 & 2.37 & 4.94 & 2.37 & 4.92 & 2.30 \\
\hline Sharing social life & 4.13 & 2.18 & 3.76 & 2.32 & 5.09 & 2.27 & 4.44 & 2.50 \\
\hline Mother to talk to me & 4.05 & 2.39 & 3.51 & 2.41 & 4.33 & 2.60 & 4.96 & 2.50 \\
\hline Parenting Centers & 4.05 & 2.32 & 4.07 & 2.44 & 4.79 & 2.44 & 4.63 & 2.43 \\
\hline Talk with my sisters & 3.90 & 2.20 & 3.42 & 2.20 & 4.46 & 2.56 & 4.77 & 2.56 \\
\hline Father to talk to me & 3.84 & 2.44 & 3.61 & 2.43 & 4.19 & 2.51 & 4.33 & 2.64 \\
\hline Get a house & 3.77 & 2.41 & 3.89 & 2.30 & 4.29 & 2.51 & 4.94 & 2.25 \\
\hline Not Rans
\end{tabular}

Note: Ranges for power to help are difficult (1) to much easier (7) and availability are strongly disagree (1) to strongly agree (7) that they can get the resource.

\section{Discussion}

This study was designed to examine factors that were associated with sexual intercourse at an early age among African born adolescents in Southern California. The theory of planned behavior was the theoretical model used to explore which constructs were important in predicting intention to have sexual intercourse at their age.

Both male and female participants in the study perceived that contracting AIDS was unlikely even if they engaged in sexual intercourse at their age, although they felt that contracting the disease was a very bad outcome. A report by HIV/AIDS and Sexual and Reproductive Health,
August, 2004 indicated a similar finding, that the risk perception among African youth is very low. The report further indicated that $62 \%$ of African adolescents who learned they were HIV positive believed they faced little or no risk at all of getting AIDS. Akwara et al. (2003) also reported that Adolescents in Africa associate the disease with homosexuals, drug users, prostitutes, truck drivers and tourists. As a result, they discount risk to themselves because they do not identify with these high-risk groups.

The females in this study seemed to be concerned about rumor spreading about them, feeling guilty and having bad reputation as likely outcomes after having sexual intercourse hence 
they thought their partners were likely to leave. But, on the other hand, they valued keeping their partners and would want to retain them in the relationships. The male respondents, on the other hand, believed that "enjoying sex" and "partner staying" after sex were the likely outcome if they had sex at their age. The results for males may suggest that African male adolescents believe they have stronger sex drives because they want to enjoy sex and keep their partners. This finding is similar to those described by Reid (1999), who found that male youth in Africa grow up with a belief that they have stronger sexual drives than women, and men cannot do without sex because they enjoy sex and take pride in having girlfriends.

It is also worth noting that even though the interaction showed that females valued the outcome "proud to be a parent", that did not positively influence their attitude toward sexual intercourse so long as they thought the outcome (being a parent) was likely. It was interesting to note that whether females thought that being a parent was an unlikely outcome or a good outcome they still felt proud to be parents and showed a more positive attitude toward sexual intercourse and may go ahead and have sex in order to become a parent. These findings in part support or concur with those of other researchers such as (Hulton et al., 2000; MacPhail \& Campbell, 2001) that African-born teens involve themselves in sex in order to have children, because having children is a proof of womanhood.

Both male and female participants thought or believed that their parents do not want them to engage in sexual intercourse at their age and they also seemed to believe that talking to parents is a powerful resource that would help them not engage in sex. It is also worth noting that on average both male and female respondents had positive thoughts or beliefs regarding availability of parental talks to them on sexual intercourse.

The average male participant felt they can talk to their fathers about sex and that fathers were both accessible and had the power to help them abstain from sex. The males also had the same trend of thought for mothers that they were available to talk about sex but they (the males) thought that mothers did not have the same power to help them avoid having sex. Compared to males, the females thought more highly about access and power about fathers talks regarding sex. These findings might suggest that African immigrant parents, particularly mothers, who have moved to the United States, do want to talk with their adolescent children about avoiding sexual intercourse at an early age, however talks from fathers may have more impact on adolescents. This finding does not concur with those of researchers such as Fitzgerald and Fitzgerald (1987) who reported that in many communities in Africa it is taboo for the children to discuss sexual matters with or in the presence of their parents, and that it is unlikely that parents will discuss sexual issues with their adolescent children. Similar findings of lack of parental guidance on sexual issues were also reported by Pratt, Quaidoo, \& Okigbo (2000) after conducting a cross sectional study to determine what social factors caused risky behaviors among Kenyan youth. It is worth noting that African adolescents recognize that parents should guide them, whether or not they are willing to comply with what the parents want them to do.

The fact that the male respondents perceived that, having a job, a house and a car might help them avoid sex but they do not have access to these resources is worth considering. Generally, many adolescents and children from poverty stricken households or war torn countries in Africa experience extreme hardships. Many are sold for sex trade because of abject poverty and they grow up believing their vulnerability is due to lack of resources. It is from such a background that a high percentage of these adolescents come and that might be one reason for such perception. This is quite different from that of adolescents from western or civilized countries where availability of such resources would enhance their involvement in sex. The majority of respondents were drawn from refugee families settled in community centers in San Diego. They were originally refugees in Kenya after they fled from other war torn countries, such as Somali, Sudan and Ethiopia. 
Lack of access to resources seemed to be an issue, which they thought, made them very vulnerable to being involved in sexual intercourse.

\section{Limitations}

This study has some limitations. Participants were from diverse African backgrounds, particularly different African countries of origin. Even for the participants from the same country some may have come from different tribal groups, and each group may have different perceptions of factors associated with sexual intercourse. There may have been subject bias where respondents gave answers according to what they perceived the researcher wanted from them. Self-reporting on sensitive issues was also likely to be a limitation because of validity biases.

A convenience sampling technique was used in conducting the survey, and therefore the findings may not be generalized to other groups or adolescents because there is no guarantee that the participants may have truly represented the underlying population who may never have had the chance or may have chosen not to participate in the study. It would be worth conducting a similar research in different parts of this country among similar populations.

\section{Conclusion and Recommendation}

The results showed that African born adolescents believed that having sex, and wanting to be a parent were important factors in their lives, even when they felt that contracting AIDS or a sexually transmitted disease would have negative consequences. The teens also indicated that talking to their parents especially their fathers would give them power to help control their sex drives. However, it is worth suggesting that programs be developed to foster parental communication on this subject with their adolescent children. This may facilitate completely decisive responses from the adolescent when another survey is conducted. On a similar note, intervention programs for adolescents should increase a sense of identification of the parents with their youth. This may positively increase the adolescents' perceptions about their parents' role in their lives regarding sexuality.

Empowerment of African born adolescents through programs that make education, information, culture and religious faith available and accessible, may significantly and positively affect their sex lives.

There may be a need to improve the socioeconomic conditions of Africa born adolescents in Southern California. This comes into focus because the survey was mostly done on those who have moved to Southern California as refugees. Since their perceptions, particularly males were on inadequate availability and access to housing, jobs, and a car; it is worth looking into their living conditions. Any intervention strategies which address issues of poverty may help in changing behavior.

\section{References}

Aggleton, P. (1996). Global priorities for HIV/AIDS intervention research. International Journal of STD and AIDS, 7(Suppl.2), 13-16.

Ajzen, I. (1991). The theory of planned behavior. Organizational Behaviors and Human Decision Processes, 50, 179-211.

Ajzen, I. (2002). Constructing a theory of planned behavior questionnaire: Conceptual and methodological considerations. Retrieved June 20, 2005, from http://www.people.umass.edu/aizen/pdf/tpb.measurement.pdf

Akwara, P. A., Madise N. J., \& Hinde, A. (2003). Perception of risk of HIV/AIDS and sexual behaviour in Kenya. Journal of Biosocial Science, 35, 285-411.

Ateka, G. (2001). Factors in HIV/AIDS transmission in Sub-Saharan Africa. Bulletin of the World Health Organization, 79, 1168.

Boer, H., \& Mashamba, M. T. (2005). Psychosocial correlates of HIV protection motivation among black adolescents in Venda, South Africa. AIDS Education and Prevention, 17, 590-602. 
Buga, G. (1996). Sexual behavior, contraceptive practice and reproductive health among school adolescents in rural Transkei. South African Medical Journal, 86 (5), 523-527.

Centers for Disease Control and Prevention (CDC). (2005a). Report on HIV/AIDS epidemic. Retrieved July 20, 2005, from http://www.cdc.gov/hiv/stats.htm\#hivest

Centers for Disease Control and Prevention (CDC). (2005b). Report on HIV/AIDS epidemic. Retrieved June 15, 2004, from http://www.cdc.gov/hiv/stats/hasrsupp.htm

County of San Diego Report. (2004). HIV/AIDS epidemiology reports December 2004. San Diego: Author.

Cook, T., \& Campbell, D. (1979). Inferring cause from passive observation. In Quasi-Experimentation design and analysis issues for field settings. Boston: Houghton Mifflin.

De Santis, J. P., \& Patsdaughter, C. A. (April, 2005). A changing course: HIV takes toll on ethnic and sexual minorities. Retrieved May 26 2005, from http://www.advanceweb.com

Didier, E. (2002, Summer). African immigrants affected by HIV. STEP perspective. Retrieved August 29, 2004, from http://www.thebody.com/step/summer01/immigrants.html

Dube, L (2005). Insights into the diffusion of HIV/AIDS information in higher education institutions in South Africa. The International Information \& Library Review, 37(4), 315-327.

Dunkle, K. L., Jewkes, R. K.; Brown, H. C.; Yoshihama, M.; Gray, G. E., \& McIntyre, J. A. et al. (2004). Prevalence and patterns of gender-based violence and revictimization among women attending antenatal clinics in Soweto, South Africa. American Journal of Epidemiology, 160, 230-239.

Erulkar, S. A., Karueru, J. P. M., Kaggwa, G., Kingola, N., Nyagah, F. K. \& Ochieng, B. (1998). Adolescents' experiences and life style in Central Province, Kenya. A baseline report. The population Council, 9-31.

Feijoo, A. N. (2004). Trends in sexual risk behaviors among high school students-United States, 1991 to 1997 and 1999 to 2003. Advocates for Youth, 4.

Fitzgerald, M., \& Fitzgerald, D. R. (1987). Parents' involvement in the sex education of their children. Volta Review, 89, 96-110.

Hartell, C. G. (2005). HIV/AIDS in South Africa: A review of sexual behavior among adolescents, University of Pretoria. Retrieved December 28, 2006, from http://www.findarticles.com/p/articles/mi_m2248/is_157_40/ai_n13774352

HIV/AIDS and Sexual and Reproductive Health. (2004, August). Reproductive health research unit. Retrieved June 7, 2005, from http://www.eldis.org/cf/search/disp/orgdisplay.cfm?org=9583\&resource=f1

Hulton, L. A., Cullen, R., \& Khalokho, W. S. (2000). Perception of risks of sexual activity and their consequences among Ugandan adolescents. Studies in Family Planning, 31, 35-46.

Joint United Nations Programme on HIV/AIDS (UNAIDS). (1998). Forces for change: World AIDS campaign with young people: 1998 world AIDS campaign briefing paper. Geneva: Author.

Joint United Nations Programme on HIV/AIDS (UNAIDS). (2000). Report on the global HIV/AIDS epidemic. Geneva: Author.

Joint United Nations Programme on HIV/AIDS (UNAIDS). (2006). 2005 report of global AIDS epidemic. Retrieved July 20, 2006, from http://www.unaids.org/bangkok2005/GAR2005

Kamya, H. A. (1997). African immigrants in the United States: The challenge for research and practice. Social Work, 42, 154-165.

Karungari, K., \& Zabin, L. (1995). Contraceptive use among high school students in Kenya. International Family Planning Perspective, 21, 108-113.

MacPhail, C., \& Campbell, C. (2001). "I think condoms are good but aai, I hate those things": Condom use among adolescents and young people in a South African township. Social Science and Medicine, 52, 1613- 1627.

Morojele, N. K., Brook, J. S., \& Kachieng’a, M. A. (2006). Perceptions of sexual risk behaviors and substance abuse among among adolescents in South Africa: A qualitative investigation [Special issue]. AIDS Care, 18, 215-219. 
Population Reference Bureau and Population Service International. (2000). Social marketing for adolescent sexual health; Results of operations research in Botswana, Cameroon, Guinea, and South Africa. Washington, DC: Author.

Pratt, C. B., Quaidoo, I. O., \& Okigbo, C. (2000). Health information sources far Kenyan adolescents: Implications for continuing HIV/AIDS control and prevention in Sub Saharan Africa. The Western Journal of Black Studies, 24(3), 131-144.

Quin, T., \& Overbough, J. (2005). HIV/AIDS in Women: An expanding epidemic. Science, 10, 15821583.

Reid, E. (1999). Placing women at the center of the analysis. United Nations Development Program (UNDP): HIV and development, 6.

Simbayi, L. C., Chauveau, J., \& Shisana, O. (2004). Behavioral responses in South Africa youth to HIV/AIDS epidemic: A nation wide survey. AIDS Care, 16 (5), 605-618.

Shisana, O. (2002). The Nelson Mandela /human science research council study on HIV/AIDS. Durban: Health System Trust. Retrieved July 24, 2006, from http://www.hsrepublishers.co.za

Summit Health Institute for Research and Education, Inc. (SHIRE). (2005, March). Giving voices to the voiceless: Language barriers \& health access issues of black immigrants of African descent. Washington, DC: Author.

\section{Acknowledgements}

I am deeply indebted to Project Bro International, Somali Youth Organization, Sudanese American Presbyterian Church and the Ethiopian Community Organization in San Diego and Orange Counties for organizing meetings with the participants. Finally, I express special gratitude to Loma Linda University and University of Eastern Africa for providing sufficient funds for conducting this project.

Author Information

Dixon Anjejo, DrPH

25526 Redlands Blvd

Loma Linda, CA, 92354

Naomi N. Modeste, DrPH, CHES*

Professor, Chair, Health Promotion and Education

Loma Linda University

School of Public Health

Loma Linda, CA, 92350

Ph. 909-558-4575

E-Mail: nmodeste@llu.edu

Jerry W. Lee, PhD, Professor

Loma Linda University

School of Public Health

Loma Linda, CA, 92350

Colwick M. Wilson, PhD

Associate Professor

Loma Linda University

School of Science and Technology

Loma Linda, CA, 92350

* corresponding author 


\section{Appendix A}

Characteristics of the study population $(\mathrm{n}=135)$

\begin{tabular}{|c|c|c|}
\hline Characteristics & $\mathbf{N}$ & $\%$ \\
\hline \multicolumn{3}{|l|}{ Age in years } \\
\hline 15 & 32 & 23.7 \\
\hline 16 & 22 & 16.3 \\
\hline 17 & 21 & 15.6 \\
\hline 18 & 36 & 26.7 \\
\hline 19 & 24 & 17.7 \\
\hline \multicolumn{3}{|l|}{ Years in S. California } \\
\hline$<1$ & 3 & 2.2 \\
\hline $1-5$ & 44 & 32.6 \\
\hline $6-10$ & 63 & 46.7 \\
\hline$>10$ & 25 & 18.5 \\
\hline \multicolumn{3}{|l|}{ Gender } \\
\hline Male & 83 & 61.5 \\
\hline Female & 52 & 38.5 \\
\hline \multicolumn{3}{|l|}{ Religion } \\
\hline Catholic & 4 & 3.0 \\
\hline Islam & 95 & 70.4 \\
\hline Protestant/Christian & 33 & 24.4 \\
\hline Other & 3 & 2.2 \\
\hline \multicolumn{3}{|l|}{ Education } \\
\hline Elementary & 1 & 0.7 \\
\hline Junior High & 8 & 5.9 \\
\hline High School & 101 & 74.8 \\
\hline College & 21 & 15.6 \\
\hline Other & 3 & 2.2 \\
\hline \multicolumn{3}{|l|}{ African Birth Country } \\
\hline Sudan & 22 & 16.3 \\
\hline Somalia & 80 & 59.3 \\
\hline Ethiopia & 26 & 19.3 \\
\hline Uganda & 1 & 0.7 \\
\hline Other & 2 & 1.5 \\
\hline Missing & 4 & 3.0 \\
\hline
\end{tabular}

